The C-Band accelerating structures for SPARC photoinjector energy upgrade

This article has been downloaded from IOPscience. Please scroll down to see the full text article. 2013 JINST 8 P05004

(http://iopscience.iop.org/1748-0221/8/05/P05004)

View the table of contents for this issue, or go to the journal homepage for more

Download details:

IP Address: 141.210.2.78

The article was downloaded on 17/06/2013 at 19:17

Please note that terms and conditions apply. 


\section{The C-Band accelerating structures for SPARC photoinjector energy upgrade}

D. Alesini, ${ }^{a, 1}$ R. Boni, ${ }^{a}$ G. Di Pirro, ${ }^{a}$ R. Di Raddo, ${ }^{a}$ M. Ferrario, ${ }^{a}$ A. Gallo, ${ }^{a}$ V. Lollo, ${ }^{a}$

F. Marcellini, ${ }^{a}$ L. Palumbo, ${ }^{b}$ V. Spizzo, ${ }^{b}$ A Mostacci, ${ }^{b}$ G. Campogiani, ${ }^{b}$

S. Persichelli, ${ }^{b}$ A. Enomoto, ${ }^{c}$ T. Higo,${ }^{c}$ K. Kakihara, ${ }^{c}$ T. Kamitani, ${ }^{c}$ S. Matsumoto, ${ }^{c}$

T. Sugimura, ${ }^{c}$ K. Yokoyama ${ }^{c}$ and S. Verdú-Andrés ${ }^{d, e}$

${ }^{a} I N F N-L N F$,

Via E. Fermi 40, 00044 Frascati, Roma, Italy

${ }^{b}$ Department of Basic and Applied Science for Engineering (SBAI) Sapienza,

University of Rome, Via A. Scarpa 14, 00161 Roma, Italy

${ }^{c}$ High Energy Accelerator Research Organization, KEK,

1-1 Oho, Tsukuba, Ibaraki 305-0801 Japan

${ }^{d}$ TERA Foundation,

Via Puccini 11, 28100 Novara, Italy

${ }^{e}$ Instituto de Física Corpuscular IFIC (CSIC-UV),

C/ Catedrático José Beltrán, 2. E-46980 Paterna, Valencia, Spain

E-mail: alesini@lnf.infn.it

ABSTRACT: The use of C-Band structures for electron acceleration and production of high quality beams has been proposed and adopted in several linac projects all over the world. The two main projects that adopted such type of structures are the Japanese Free Electron Laser (FEL) project in Spring-8 and the SwissFEL project at Paul Scherrer Institute (PSI). Also the energy upgrade of the SPARC photo-injector at LNF-INFN (Italy) from 150 to more than $240 \mathrm{MeV}$ will be done by replacing a low gradient $\mathrm{S}$-Band accelerating structure with two C-band structures. The structures are Traveling Wave (TW) and Constant Impedance (CI), have symmetric axial input couplers and have been optimized to work with a SLED RF input pulse. The paper presents the design criteria of the structures, the realization procedure and the low and high power RF test results on a prototype. The high power tests have been carried out by the Frascati INFN Laboratories in close collaboration with the Japanese Laboratory KEK. Experimental results confirmed the feasibility of the operation of the prototype at $50 \mathrm{MV} / \mathrm{m}$ with about $10^{6}$ breakdowns per pulse per meter. Such high gradients have not been reached before in C-Band systems and demonstrated the possibility to use C-band accelerators, if needed, at such high field level. The results of the internal inspection of the structure after the high power test are also presented.

\footnotetext{
${ }^{1}$ Corresponding author.
} 
KEYWORDS: Acceleration cavities and magnets superconducting (high-temperature superconductor; radiation hardened magnets; normal-conducting; permanent magnet devices; wigglers and undulators); Accelerator Subsystems and Technologies; Instrumentation for FEL 


\section{Contents}

1 Introduction 1

2 Structure design $\quad 3$

$\begin{array}{lll}3 & \text { Structure fabrication } & 7\end{array}$

4 RF test $\quad 8$

4.1 High-power test 9

5 Structure internal inspection after high power test 12

6 Conclusions 12

A Calculation of figures of merit of the C-band single cell 14

B Calculation of the structure parameters with a SLED-type input pulse 16

C Analysis of the multipolar field components introduced by the couplers 17

\section{Introduction}

The use of C-Band structures for electron acceleration and production of high quality beams has been proposed and adopted in several FEL projects all over the world [1-6]. It has also been proposed and successfully tested in the injector linac of the electron-positron collider SuperKEKB [7, 8] The two main projects that adopted C-band structures are the Japanese FEL project $[1,2]$ in Spring-8 and the SwissFEL project at PSI [5]. In the first project travelling wave (TW) damped structures have been adopted for multi-bunch operation [4, 9-14]. The damping of the High Order Modes (HOM) is realized with the choke mode technique. The structures, operating with a $3 \pi / 4$ phase advance per cell, were successfully tested with accelerating gradients of $37 \mathrm{MV} / \mathrm{m}$ [15]. In the PSI project the structures have been designed as constant gradient and will operate at an average gradient of $26 \mathrm{MV} / \mathrm{m}$ [5]. High power test on short prototypes have been recently done giving gradients $>40-50 \mathrm{MV} / \mathrm{m}[39]$.

In the SPARC photoinjector the choice of the C-Band for the energy upgrade [16] was dictated by the opportunity to achieve a higher accelerating gradient, enabled by the higher frequency and to explore a C-band acceleration combined with an S-band injector that, at least from beam dynamics simulations [17] was very promising in terms of achievable beam quality.

SPARC is a $150 \mathrm{MeV}$ photoinjector test facility in operation since 2001 in the Laboratories of the National Institute of Nuclear physics in Frascati (LNF-INFN, Italy) [18]. The photoinjector has 


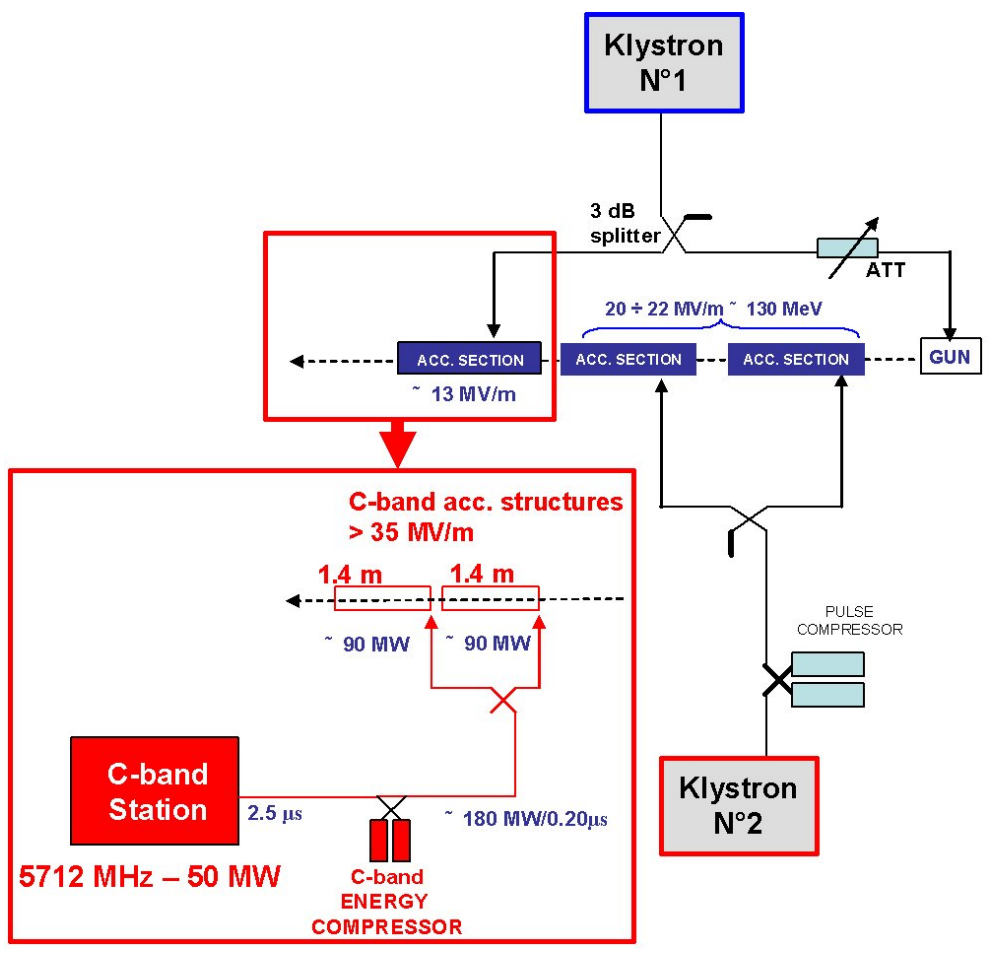

Figure 1. Sketch of the SPARC layout after with the C-band system installation.

been constructed to produce high brightness electron beams for several experiments like SASEFEL in visible light [19], velocity bunching [20] and seeding [21]. The beam is generated in a 1.6 cell standing wave RF gun of the UCLA/BNL/SLAC type [22] and accelerated with three constant gradient $2 \pi / 3 \mathrm{TW}$ structures. All systems operate in S-Band at $2.856 \mathrm{GHz}$.

The upgrade of the beam energy of the photoinjector from $15 \mathrm{MeV}$ to more than $24 \mathrm{MeV}$, will be done by replacing the last $3 \mathrm{~m}$ long low gradient $(<13 \mathrm{MV} / \mathrm{m}) \mathrm{S}$-band TW accelerating section with two $1.4 \mathrm{~m}$ long structures operating in the C-band (i.e., at $5712 \mathrm{MHz}$ ), as shown in figure 1.

The new C-band structures will be fed by a Toshiba ET37202 50-MW C-band klystron. The HV pulsed modulator and the $400 \mathrm{~W}$ solid state driver for the klystron were manufactured by ScandiNova (Sweden) and Mitec Telecom (Canada), respectively, and have already been installed and tested at LNF. The new C-band system will also include a SKIP-type pulse compressor [23] that is presently under construction. The C-band structures have been developed in the LNF-INFN laboratories with the support of local firms for their construction. To simplify the electromagnetic and mechanical design, we chose a constant impedance (CI) structure with large irises that limits the surface electric field and allow a larger pumping speed. In addition they also allow increasing the group velocity and, consequently, allow feeding the unit with shorter RF pulses over a short filling time. It must be noted that, with the use of pulse compressors, the decay of the RF pulse amplitude along a CI structure is compensated by the exponential shape of the SLED pulse, resulting in a rather constant profile of the RF field along the accelerating section, as discussed in appendix B.

An important assumption to support the above mentioned upgrade is that an average acceleration field of more than $35 \mathrm{MV} / \mathrm{m}$ will be obtained. This would result in a total energy gain of more 
than $90 \mathrm{MeV}$ with the two C-band sections. To verify the validity of all technical choices, a prototype consisting of $20 \mathrm{RF}$ cells and having a total length of $50 \mathrm{~cm}$ was designed, built, and brazed at LNF. The prototype has been high-power tested in Japan at KEK, the High Energy Accelerator Research Organization, where a power station of $50 \mathrm{MW}$ at $5712 \mathrm{MHz}$ is available. This was done within the framework of a collaboration established ad hoc between KEK and INFN.

In the first section of the paper we summarize the design criteria adopted for the structures. In the second one we give some detail on the mechanical fabrication of the prototype while in the third section we will illustrate the RF experimental measurements and the high power test results. The analysis of the structure after the high power test by cutting the cells is shown in the fourth section. Conclusions and perspectives are finally reported

\section{Structure design}

The design criteria of the C-band structures are illustrated and discussed in detail in [24]. The structures are TW CI sections with symmetric axial input couplers and have been optimized to be fed with a SLED-type RF input pulse. The SPARC linac operates in single bunch mode and the choice of a CI structure was made partly to reduce the fabrication costs but mainly to obtain a quasi-uniform accelerating field along the structure when the structure is fed by the SLED RF pulse. The decay of the RF pulse amplitude along a CI structure is, in fact, compensated by the exponential shape of the SLED pulse, resulting in a rather constant profile of the RF field along the accelerating section.

The mechanical drawings of the structure and of the prototype are given in figure 2 .

The final dimensions and shape of the single cell (reported in figure 3) were optimized to obtain:

1. the lowest peak electric field on the iris surfaces;

2. an average accelerating field of (at least) $35 \mathrm{MV} / \mathrm{m}$ with the power available from the klystron combined with the SLED;

3. the largest possible iris aperture, compatible with the points above, in order to increase the pumping speed and thereby reduce the wake field contribution as well as the filling time of the structure itself. A short filling time reduces the required input pulse length. Shorter pulses are preferred as RF breakdown probability increases with pulse length [25].

In the optimization procedure the main figures of merit (shunt impedance r, field attenuation $\alpha$, ratio between the average accelerating field and the surface field $E_{\mathrm{s} \text { peak }} / \mathrm{E}_{\mathrm{acc} \_\mathrm{av}}$, group velocity $\mathrm{v}_{\mathrm{g}}$ ) have been calculated as a function of the iris radius (a) and thickness ( $t$ ). An elliptical shape of the iris profile has been also adopted to further reduce the surface field. The details of the analysis are summarized in appendix A. The previous quantities have been combined with the SLED-type RF pulse in order to calculate the average accelerating field $\left(\mathrm{E}_{\mathrm{acc} \_a v}\right)$, the peak surface field $\left(\mathrm{E}_{s}\right.$ peak $)$ and the uniformity of the accelerating field along the structure. The final results are given in figure 4 . The peak surface field has been calculated in two different conditions. The first one is related to the case of the maximum input power that we considered reasonably available from the source, the 


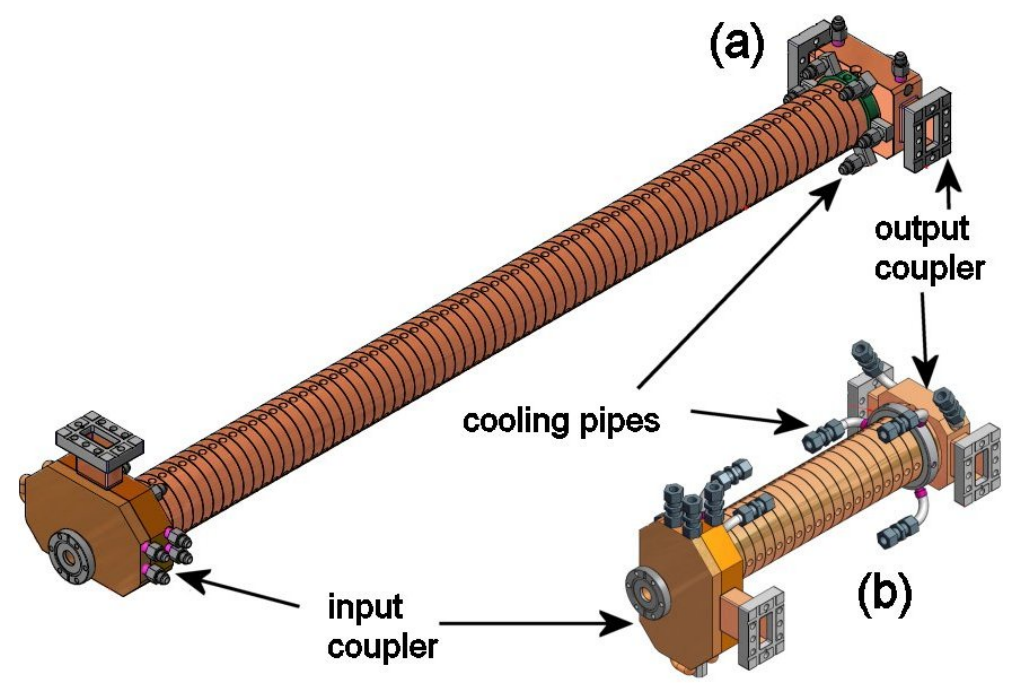

Figure 2. Mechanical drawing of the C-Band Structure (a) and prototype (b).

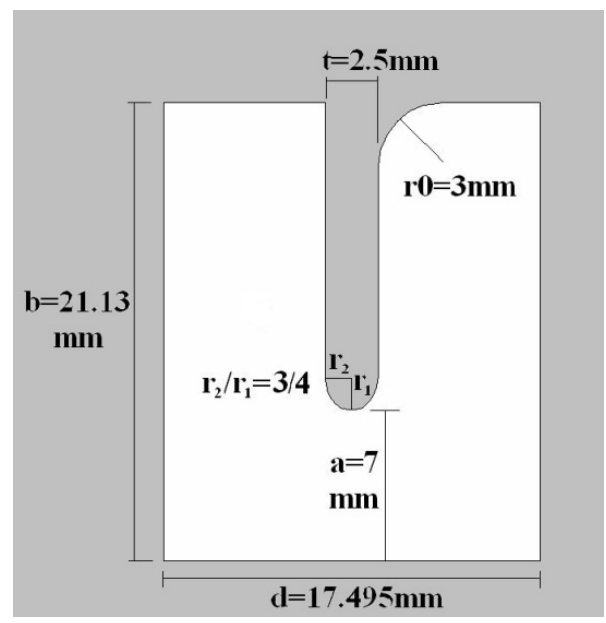

Figure 3. Dimensions of the single cell.

second one in the case of an average accelerating field of $35 \mathrm{MV} / \mathrm{m}$. The detail of the calculations is summarized in appendix B.

Following the design criteria illustrated at the beginning of this section and the results shown in figure 4, we decided to adopt an iris radius of $7 \mathrm{~mm}$ with $2.5 \mathrm{~mm}$ thickness and an elliptical shape of the iris profile. In the final cell profile we have decided to round one sharp edge of the cell outer profile, in order to increase the quality factor as much as possible. This type of shape is compatible with the cell machining, as illustrated in the next section. The final structure parameters are given in table 1 . They are referred to the final $1.4 \mathrm{~m}$ long structure and to the prototype we have built for high power tests.

The sensitivity of the phase advance per cell as a function of the geometrical parameters is finally shown in table 2. From these quantities it is possible to extrapolate the maximum tolerances of fabrication and how to correct errors with a proper cell tuning. 
Table 1. Final structure parameters (HFSS results).

\begin{tabular}{|c|c|c|}
\hline Parameter & Prototype & Final structure \\
\hline Frequency $\left(f_{R F}\right)$ & \multicolumn{2}{|c|}{$5.712[\mathrm{GHz}]$} \\
\hline Phase advance per cell & \multicolumn{2}{|c|}{$2 \pi / 3$} \\
\hline Number of accelerating cells $(\mathrm{N})$ & 22 & 71 \\
\hline Structure length including couplers (L) & $0.54[\mathrm{~m}]$ & $1.4[\mathrm{~m}]$ \\
\hline Cell length $(\mathrm{d})$ & \multicolumn{2}{|c|}{$17.495[\mathrm{~mm}]$} \\
\hline Iris radius (a) & \multicolumn{2}{|c|}{$7[\mathrm{~mm}]$} \\
\hline Iris thickness $(\mathrm{t})$ & \multicolumn{2}{|c|}{$2.5[\mathrm{~mm}]$} \\
\hline Outer radius $(b)$ & \multicolumn{2}{|c|}{$21.13[\mathrm{~mm}]$} \\
\hline Group velocity $\left(\mathrm{v}_{\mathrm{g}} / \mathrm{c}\right)$ : & \multicolumn{2}{|c|}{0.0283} \\
\hline Field attenuation $(\alpha)$ & \multicolumn{2}{|c|}{$0.206[1 / \mathrm{m}]$} \\
\hline Series impedance $(Z)$ & \multicolumn{2}{|c|}{$34.1\left[\mathrm{M} \Omega / \mathrm{m}^{2}\right]$} \\
\hline Shunt impedance (r) & \multicolumn{2}{|c|}{$82.8[\mathrm{M} \Omega / \mathrm{m}]$} \\
\hline Filling time $\left(\tau_{F}\right)$ & $50[\mathrm{~ns}]$ & $150[\mathrm{~ns}]$ \\
\hline $\mathrm{E}_{\mathrm{s} \text { peak }} / \mathrm{E}_{\mathrm{acc} \_\mathrm{av}}$ & \multicolumn{2}{|c|}{2.17} \\
\hline $\mathrm{H}_{\text {s peak }} / \mathrm{E}_{\mathrm{acc} \_a v}$ & \multicolumn{2}{|c|}{$2.49[\mathrm{kA} / \mathrm{MV}]$} \\
\hline$\sqrt{S_{\mathrm{c}, \max }} / \mathrm{E}_{\mathrm{acc} \_\mathrm{av}}{ }^{1}$ & \multicolumn{2}{|c|}{$0.024 \sqrt{\mathrm{MW} / \mathrm{mm}^{2}} / \mathrm{MV} / \mathrm{m}$} \\
\hline Pulsed heating $\mathrm{E}_{\mathrm{acc} \_\mathrm{av}}=35 \mathrm{MV} / \mathrm{m}$ & \multicolumn{2}{|c|}{$<1^{\circ} \mathrm{C}$} \\
\hline $\begin{array}{l}\text { Maximum average accelerating gradient } \\
\text { with the available klystron power and } \\
\text { SLED }\end{array}$ & $60.2[\mathrm{MV} / \mathrm{m}]$ & $51.6[\mathrm{MV} / \mathrm{m}]$ \\
\hline $\begin{array}{l}\text { Peak surface field (at the beginning of } \\
\text { the SLED pulse for the maximum power } \\
\left.\text { level (and scaled for } E_{\text {acc_av }}=35 \mathrm{MV} / \mathrm{m}\right) \text { ) }\end{array}$ & \multicolumn{2}{|c|}{$140(96)[\mathrm{MV} / \mathrm{m}]$} \\
\hline $\begin{array}{l}\text { Maximum energy gain (and at } \mathrm{E}_{\mathrm{acc} \_a v}= \\
35 \mathrm{MV} / \mathrm{m} \text { ) }\end{array}$ & $23.2(13.5)[\mathrm{MeV}]$ & $64.1(43.5)[\mathrm{MeV}]$ \\
\hline Output power & $0.85 \cdot \mathrm{P}_{\text {in }}$ & $0.60 \cdot \mathrm{P}_{\text {in }}$ \\
\hline Average dissipated power $10 \mathrm{~Hz}$ rep. rate & $7.6[\mathrm{~W}]$ & $59.6[\mathrm{~W}]$ \\
\hline
\end{tabular}

The adopted input and output couplers are the so-called "waveguide-type" couplers [2628]. This type of coupler allows obtaining low pulsed heating and compact symmetric feeding simultaneously.

\footnotetext{
${ }^{1} S_{\mathrm{c}, \max }$ is the maximum modified Poynting vector.
} 
Table 2. Sensitivity of the phase advance per cell as a function of the geometrical parameters.

\begin{tabular}{|c|c|c|}
\hline Parameter & $\begin{array}{c}\text { Cell phase advance Sensitivity } \\
(\mathrm{deg} / \mu \mathrm{m})\end{array}$ & $\begin{array}{c}\text { Frequency Sensitivity } \\
(\mathrm{kHz} / \mu \mathrm{m})\end{array}$ \\
\hline $\mathrm{b}$ & 0.22 & -291 \\
\hline $\mathrm{a}$ & -0.062 & 81 \\
\hline $\mathrm{t}$ & -0.012 & 16 \\
\hline $\mathrm{d}$ & 0.0087 & -11 \\
\hline
\end{tabular}
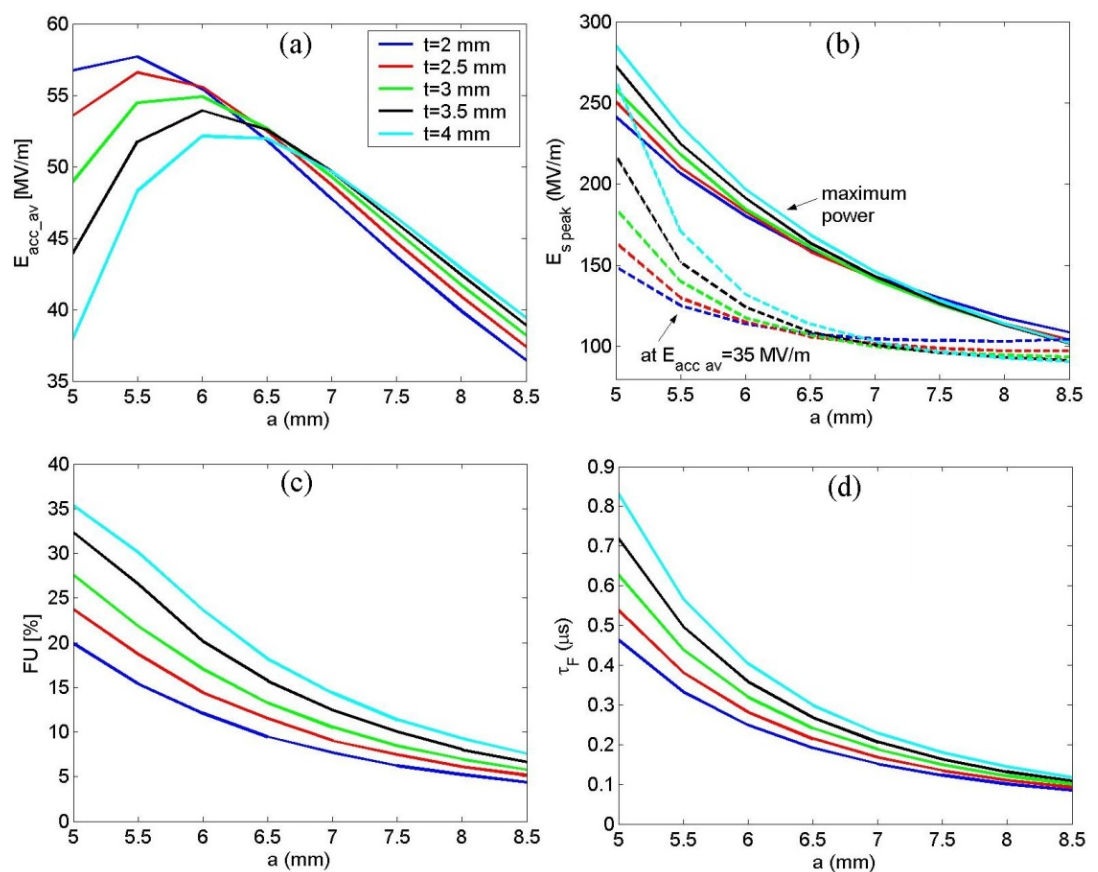

Figure 4. Main structure figures of merit as a function of the iris aperture and thickness: (a) average accelerating field; (b) peak surface field at the beginning of the RF pulse; (c) accelerating Field Uniformity along the structure; (d) filling time.

The sketch of the complete input coupler with few TW cells is given in figure 5(a). The design has been divided into two parts, the coupler and the splitter. The first one has been designed by tuning the coupling iris and the outer radius of the first cell (shown in figure 5(b)) to match the waveguide mode $\mathrm{TE}_{10}$ with the accelerating mode $\mathrm{TM}_{01}$-like. This design has been done using HFSS and following the procedure illustrated in [29]. The second one has been done by an optimization procedure of the splitter internal shape and dimensions to minimize the reflection coefficient at the input port (figure 5(c)). The final obtained reflection coefficient was $<-40 \mathrm{~dB}$. As an example the amplitude and phase of the accelerating field and the accelerating field "seen" by a particle on crest are given in figure 6 for a 3 cell plus in/out couplers structure.

Concerning the peak magnetic field (and the related peak pulsed heating) on the coupler cell iris, it is lower than that in the regular cell.

Field multi-polar components introduced by the waveguide coupler were investigated during the design phase. The coupler, as a matter of fact, is a compact transition between a rectangular 


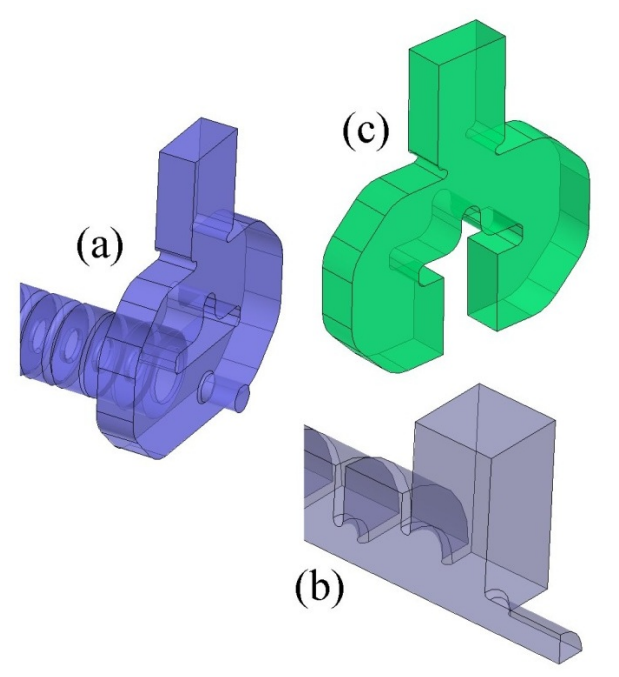

Figure 5. (a) Sketch of the complete coupler with few TW cells; (b) detail of the waveguide coupler; (c) detail of the splitter.

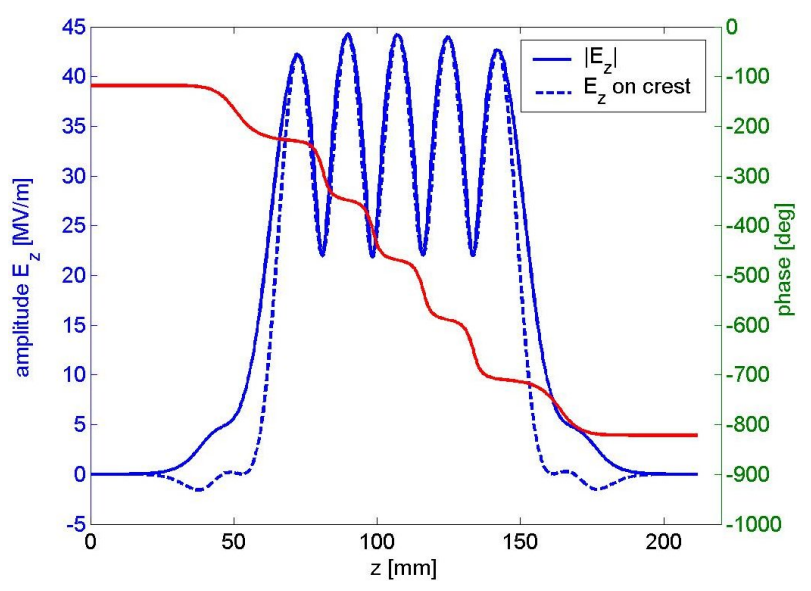

Figure 6. Amplitude and phase of the accelerating field ( 3 cells plus in/out couplers structure) and accelerating field "seen" by a particle on crest.

geometry (the waveguide) and a circular one and, therefore, it introduces multi-polar field components in the waveguide region. Due to the symmetric feed of the system these components have an even periodicity with respect to the azimuthal angle. The details of these analysis are reported in appendix C.

Also dipole components of the field in the input couplers can occur if there are unbalances between the phase in the two harms of the integrated power splitter. We have calculated this components and the results are given in appendix $\mathrm{C}$.

\section{Structure fabrication}

The picture of a single cell is shown in figure 7(a). Each cell was machined as a cup to include a single iris. The integrated cooling system employs six longitudinal cooling pipes. Three tuners are inserted at $120^{\circ}$ to one another, and these can deform the outer wall of each cell in both directions. 

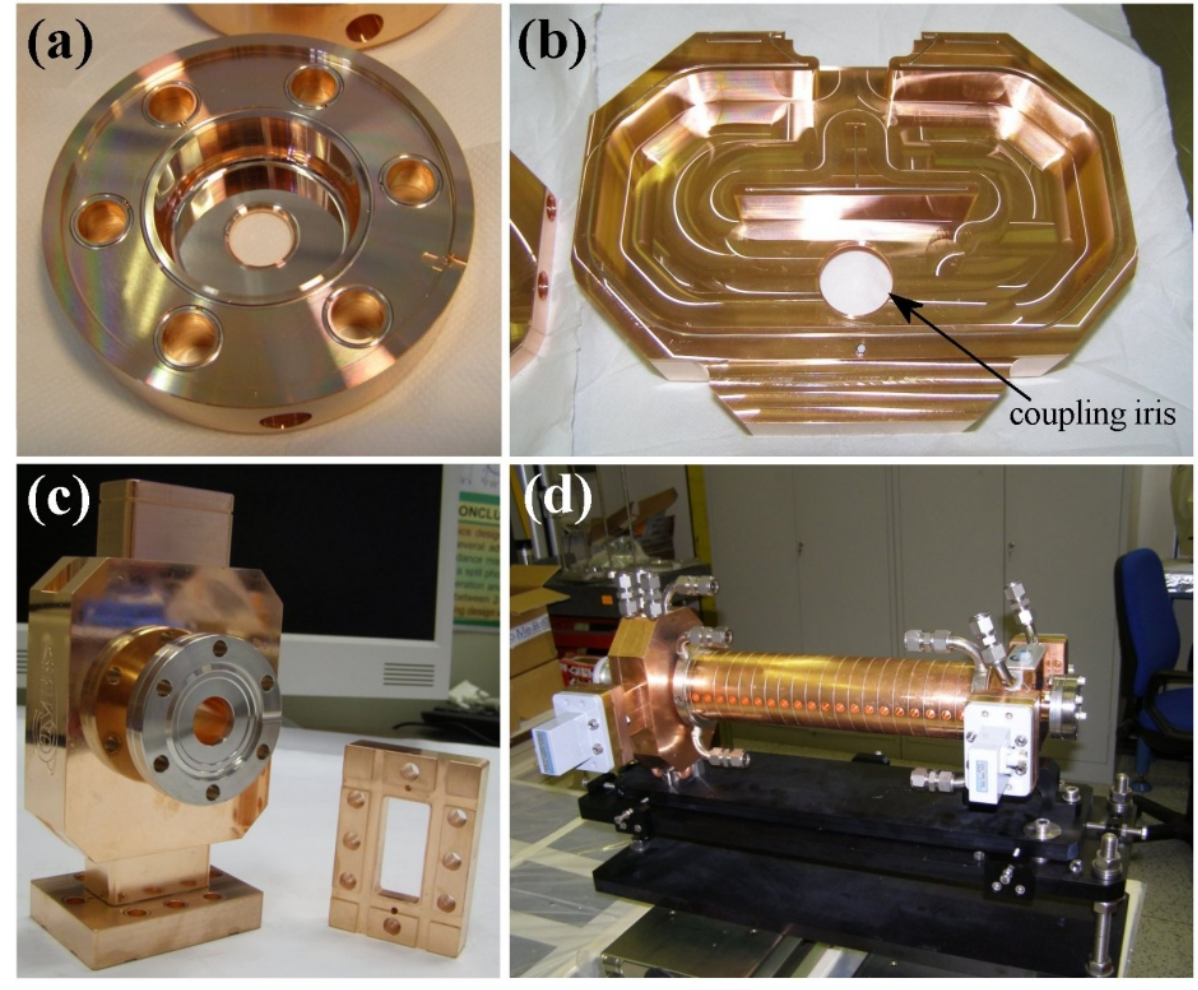

Figure 7. (a) Regular cell; (b) input coupler; (c) output coupler and (d) final prototype.

Cells were machined by the local firm Comeb [30] using a high-precision turning machine. The obtained surface roughness was less than $50 \mathrm{~nm}$, and the precision in the dimensions was $\pm 2 \mu \mathrm{m}$.

The input coupler was fabricated by milling a single copper ingot with a computer-controlled machine and then was closed with a blanking plate brazed onto the coupler itself, together with the input flange. The obtained surface roughness was less than $200 \mathrm{~nm}$, and the precision in all the dimensions was $\pm 10 \mu \mathrm{m}$. A picture of the input coupler is shown in figure 7(b).

Before the brazing of the cells, they were cleaned with a detergent (ALMECO-19) and a mixture of organic (citric) acid and distilled water, in a bath with an ultra-sound machine.

To minimize the number of brazing processes and increase the speed of manufacturing, the inner surface of both rectangular output couplers was prepared by the electric discharge machining (EDM) technique. However, the coupling irises and the pipe were fabricated using a milling machine. The obtained surface roughness of the waveguide was $0.6 \mu \mathrm{m}$, and the precision in the dimensions was $\pm 20 \mu \mathrm{m}$. A picture of the output coupler is shown in figure 7(c).

The structure was brazed at LNF in a vacuum furnace through various steps. The final prototype structure is given in figure $7(\mathrm{~d})$.

\section{RF test}

RF measurements were conducted at each brazing step and after the final brazing process. Figure 8 shows the reflection coefficient at the structure input port after the final brazing before and after the tuning. We also measured the longitudinal electric field along the structure (using the bead-pull 


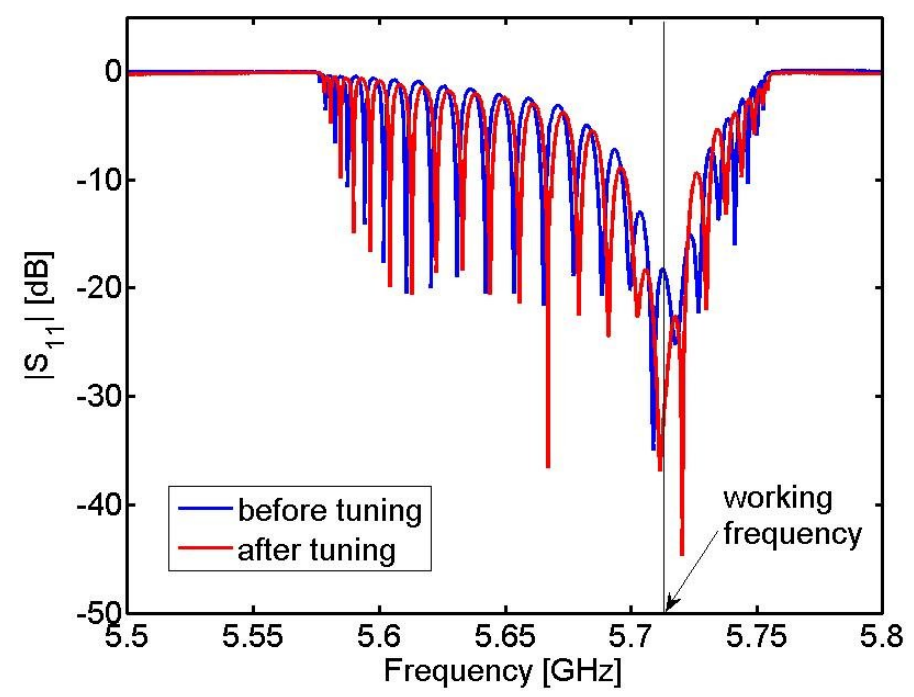

Figure 8. Measured reflection coefficient at the structure input port before and after the tuning.
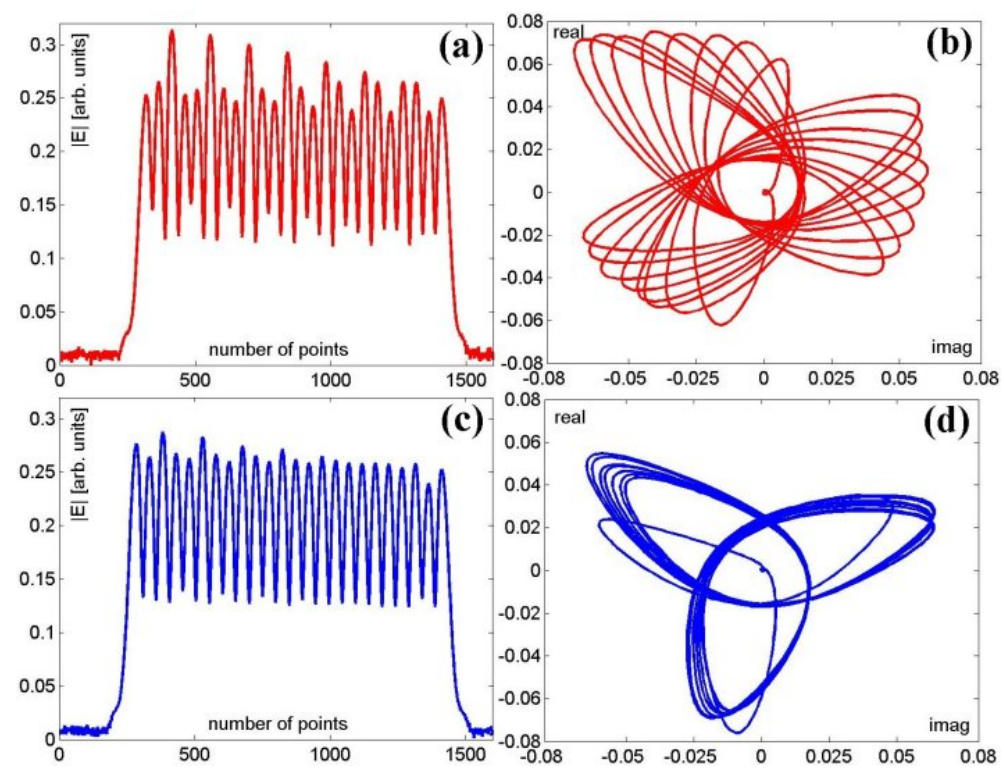

Figure 9. Measured longitudinal electric field on axis before (a)-(b) and after (c)-(d) tuning.

technique [31]), and the result is shown in figure 9. The tuning of the structure has been done following the procedure reported in [32] with some modifications and is widely illustrated in [33]. The measured reflection coefficient after tuning was $<-30 \mathrm{~dB}$. A residual detuning of the structure (and therefore a field flatness unbalance) is still visible and it was not possible to compensate it. Possible reasons of these small residual errors are discussed in [33] and are still under discussion.

\subsection{High-power test}

A detailed description of the high power test and results is given in [34]. The C-band test area at KEK is mainly composed of a klystron driven by a PFN modulator, a $22 \mathrm{~m}$ long low loss transport line, a SLED-type pulse compressor and a concrete vault of test area. The picture of the structure 

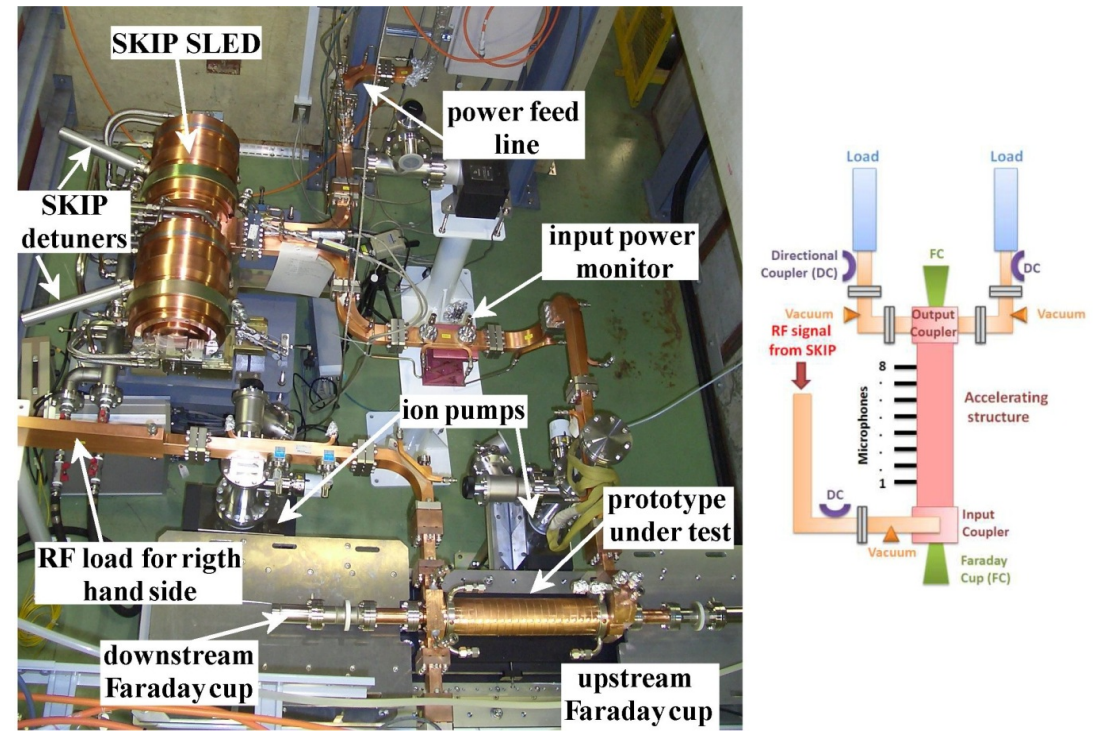

Figure 10. Experimental setup inside the shielded room and its schematic setup.

prototype under test in the radiation shield room is given in figure 10. The power from the klystron is transported by a low-loss waveguide to the roof of the concrete vault. It is converted to the $\mathrm{TE}_{10}$ mode in the rectangular waveguide by a mode converter and then admitted into the shielded room. The power is pulse compressed by a SKIP-type SLED [23], when needed, before feeding the accelerator structure. Evacuation was performed by ion pumps through waveguide evacuation ports, one in front of the input coupler and two in the output waveguides between the accelerator structure and RF loads. The input power was monitored by the directional coupler located between SKIP and the accelerator structure. The transmitted power was monitored in front of each RF load. An array of acoustic sensors were mounted on the test structure and various parts of the waveguide. The currents emitted from the accelerator structure were monitored in both directions. Pipes with an inner diameter of $40 \mathrm{~mm}$ were connected from the beam ports of the accelerator structure with ceramic insulation in front of the Faraday cups

The high-power test started on November 5, 2010 and was completed on December 13, 2010. For almost one month of processing, from November 5 until December 2, more than $10^{8} \mathrm{RF}$ pulses of $200 \mathrm{~ns}$ width were sent into the structure with a repetition rate of $50 \mathrm{~Hz}$. For a couple of days the RF pulse length was changed to $300 \mathrm{~ns}$ and for one day (November 12) the repetition rate was decreased to $25 \mathrm{~Hz}$. On November 15, SKIP was switched on. After that, the power $P_{\text {in }}$ into the structure was about five times the power $\mathrm{P}_{\mathrm{F}}$ delivered by the klystron. Figure 11 shows the full test history in terms of the klystron output power $\mathrm{P}_{\mathrm{K}}$.

The dark current was measured for different pulse lengths (from 100 to $1600 \mathrm{~ns}$ ) and different field values. The measured values reported in figure 12 show, as example, the linear dependence of the dark current on the RF pulse length.

The breakdown rate (BDR) is defined in the present paper by the breakdowns per pulse per meter $(\mathrm{bpp} / \mathrm{m})$ and is calculated as follows:

$$
\mathrm{BDR}=\frac{\text { number of breakdowns }}{\mathrm{t}_{\text {meas }} \cdot \mathrm{PRF} \cdot \mathrm{L}_{\text {struct }}}[\mathrm{BD} / \text { pulse } / \mathrm{m}]
$$




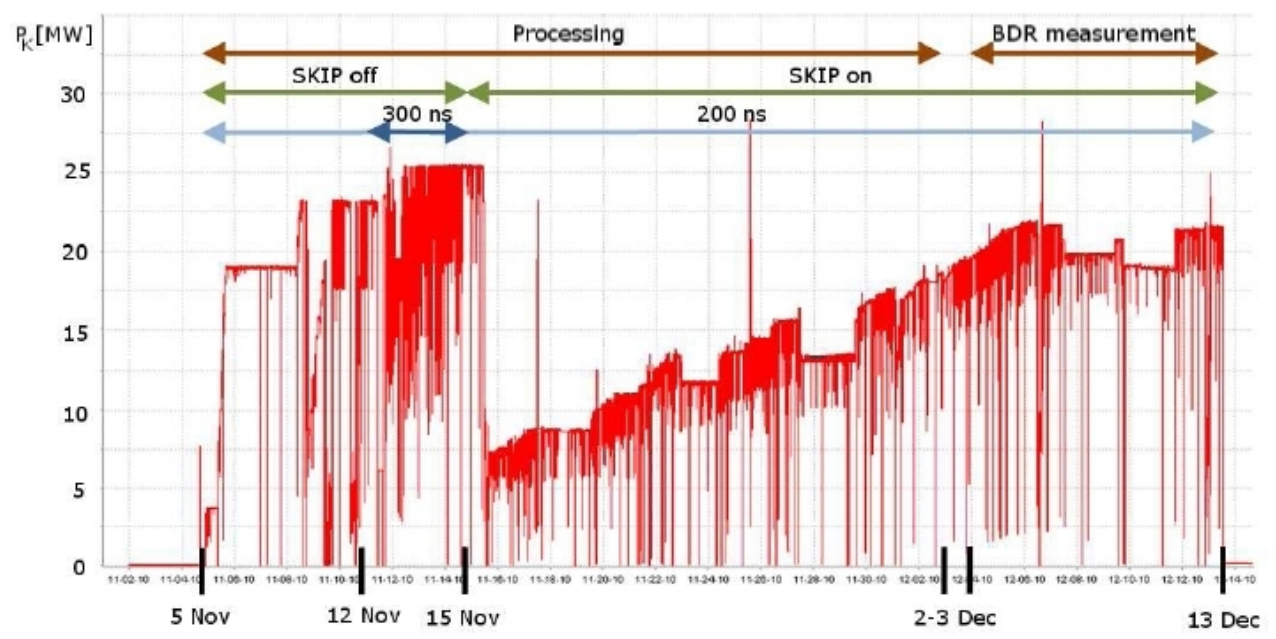

Figure 11. History of high-power test of structure.

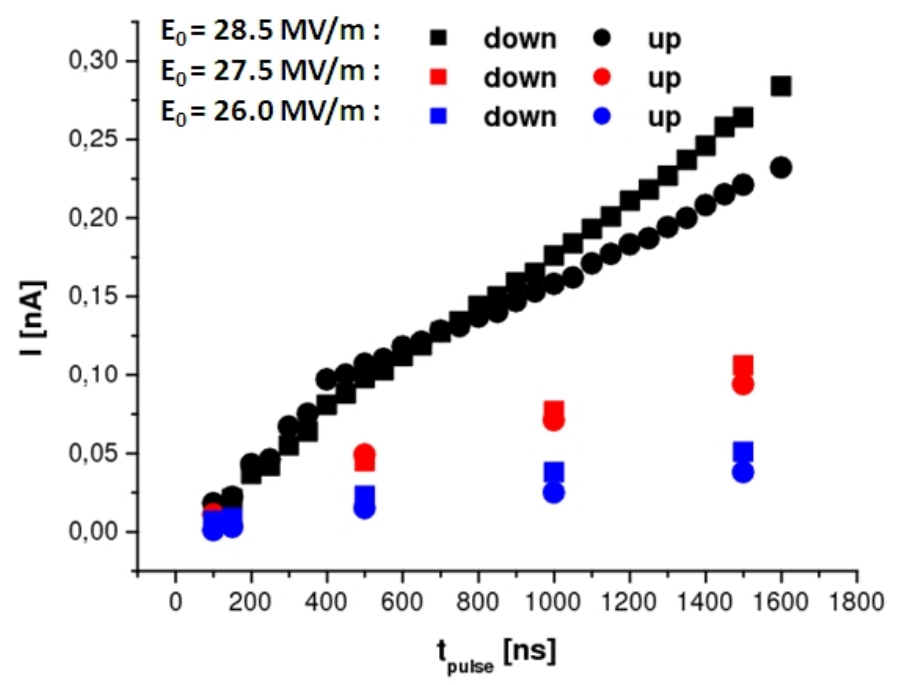

Figure 12. Dependence of dark current on RF pulse length for different accelerating field values.

where $t_{\text {meas }}$ is the period of the measurement, PRF is the repetition rate, and $\mathrm{L}_{\text {struct }}$ is the length of the test structure $(0.35 \mathrm{~m})$ Breakdowns were detected from the field-emission current signal

The dependence of the breakdown rate on the accelerating gradient is given in figure 13. The blue squares correspond to the BDR measurements performed during the structure conditioning and the red circles to those performed after the structure was conditioned. As shown in the figure, the performance of the structure was significantly improved by conditioning and BDR of few $10^{-6}$ $\mathrm{bpp} / \mathrm{m}$ have been finally reached at accelerating gradients above $50 \mathrm{MV} / \mathrm{m}$.

We have tried to calculate the distribution of breakdown events along the TW structure following two different approaches: through the analysis of transmitted and reflected RF pulses during the breakdown phenomena and analyzing the signals form acoustic sensors. The detail of such analysis is given in [34] and has shown a different localization in the two cases. From the first analysis the main source of breakdown has been localized in the output couplers and adjacent cells 


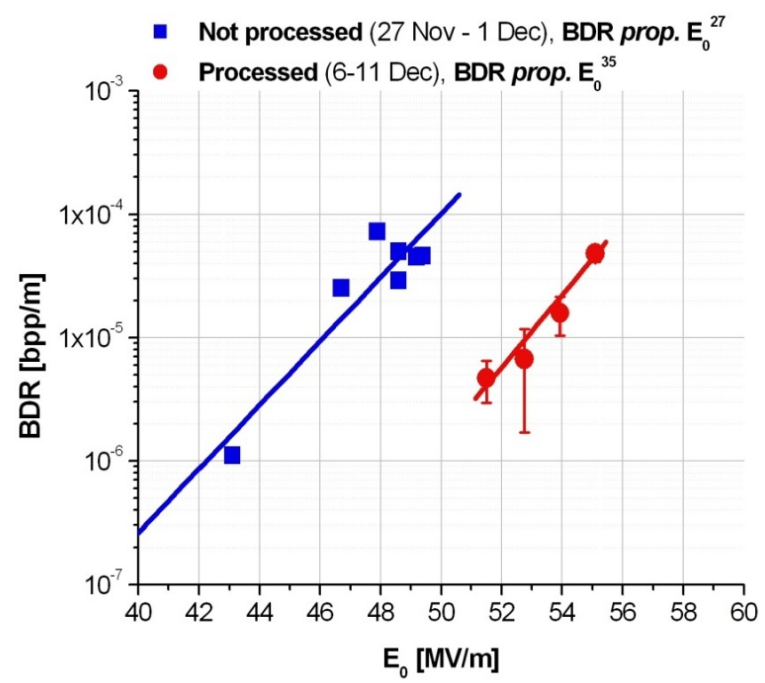

Figure 13. Breakdown rate at different accelerating field values before and after conditioning.

while for the second type mainly in the first accelerating cells. After the internal inspection of the structure (described in the next paragraph) the second type of analysis has been confirmed to be more reliable.

\section{Structure internal inspection after high power test}

After the high power test the structure has been cut in slices for an internal inspection. Figure 14 shows the lines of cutting and the adopted classification of the cells. We have identified the signs of craters and discharges mainly in the first accelerating cell after the input coupler, as expected, because the highest field values are excited at the beginning of CI structures. The surface of this cell is shown in figure 15(a). A micro-analysis with the SEM has been also done. Figure 15(b) reports the image of one of the craters due to breakdowns. With the micro-analysis we have also seen a contamination of $\mathrm{C}, \mathrm{Al}, \mathrm{Mg}$ and $\mathrm{Si}$ inside the craters that is probably related to the presence of dust micro-particles. These particles probably drove the discharges. In any central cell no pits or discharges were visible. As an example in figure 16 is shown cell number 6 where the crystals growth of the copper due to the high temperature brazing process is clearly visible. No signs of breakdown or damages have been seen in the input and output couplers.

In conclusion the most discharges occurred in the first accelerating cell, as expected. From this results the calculation of breakdown location with acoustic sensors was more reliable than the analysis by RF signals.

\section{Conclusions}

Two $1.4 \mathrm{~m}$ long C-band TW constant-impedance structures will be installed in the SPARC FEL for the beam energy upgrade from 150 to more than $240 \mathrm{MeV}$. In the paper we illustrated the design criteria of these structures. A prototype with a reduced number of cells has been fabricated and tested at high power at KEK giving very good performances in terms of breakdown rates at high accelerating gradient $\left(>50 \mathrm{MV} / \mathrm{m}\right.$ with $\mathrm{bpp} / \mathrm{m} \sim 10^{-6}$ ). Detail of the fabrication process, tuning 


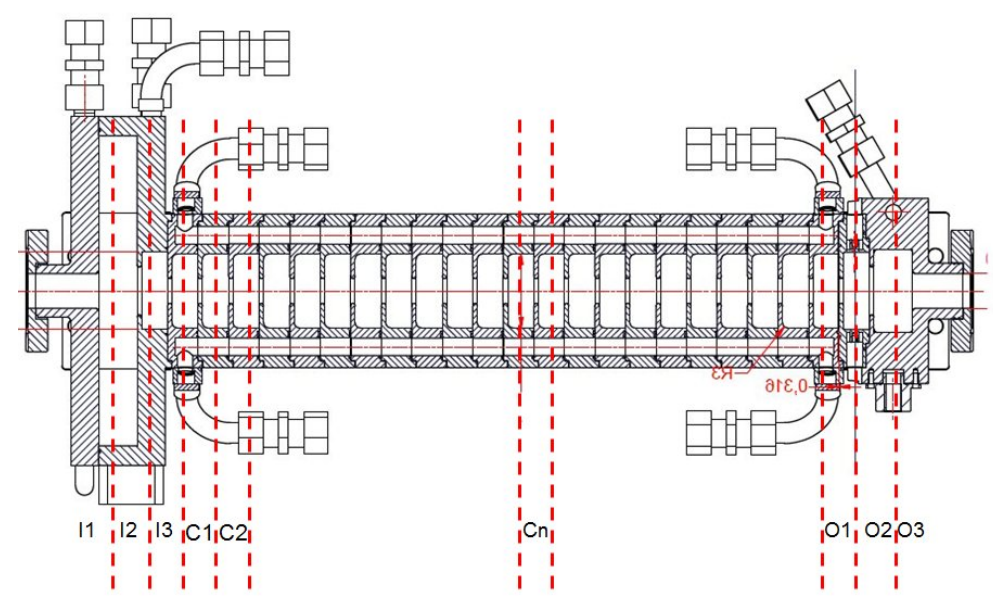

Figure 14. Lines of cutting of the prototype after high power tests.

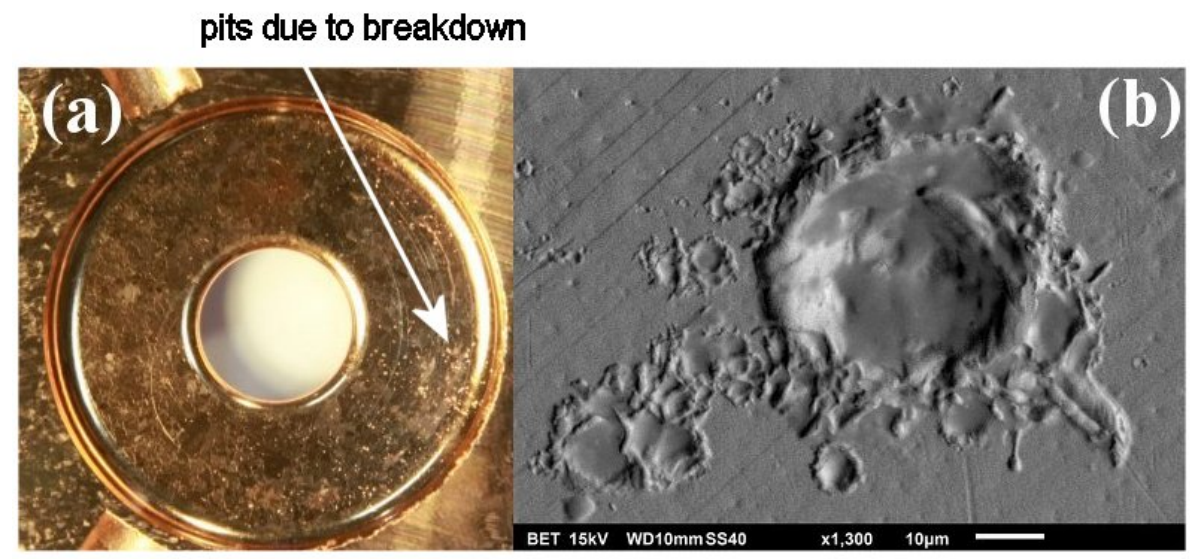

Figure 15. (a) picture of the first accelerating cell, (b) SEM image of craters due to breakdown.

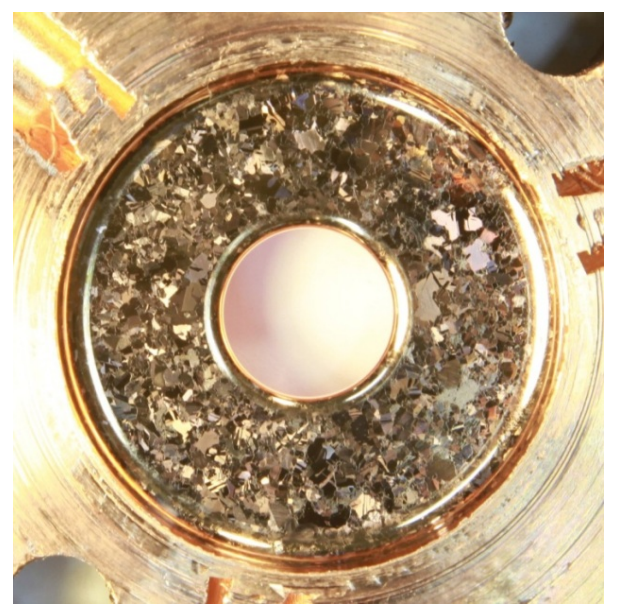

Figure 16. Picture of accelerating cell number 6. 
procedure and high power RF test results have been presented and discussed.The high-power tests of the C-band prototype carried out at KEK have confirmed the validity of the technical choices and the feasibility to use C-band accelerators, as needed, at such a field level

\section{Acknowledgments}

Special thanks are due to the Frascati technical staff (A. Battisti, R. Clementi and S. Quaglia) for its precious support during the test at KEK and to R. Zennaro and A. Citterio for their help in the tuning of the structure. The research leading to these results has received funding from the European Commission under the FP7-INFRASTRUCTURES-2010-1/INFRA-2010-2.2.11 project TIARA (CNI-PP) Grant agreement no 261905 and by the Seventh Framework Programme [FP7/20072013] under grant agreement no. 215840-2.

\section{A Calculation of figures of merit of the C-band single cell}

The sketch of the single cell with its main dimensions is given in figure 17.

The main figures of merit for a single cell are given below:

$$
\begin{aligned}
Z & =\frac{\mathrm{E}_{\text {acc }}^{2}}{\mathrm{P}_{\text {in }}} & & \text { series impedance } \\
\alpha & =\frac{\mathrm{p}_{\text {diss }}}{2 \mathrm{P}_{\text {in }}} & & \text { field attenuation constant } \\
v_{g} & =\frac{\mathrm{P}_{\text {in }}}{w} & & \text { group velocity } \\
r & =\frac{\mathrm{E}_{\text {acc }}^{2}}{\mathrm{p}_{\text {diss }}} & & \text { shunt impedance per unit length }
\end{aligned}
$$

where:

$$
\begin{array}{rlrl}
e V_{z} & =e\left|\int_{0}^{D} \mathrm{E}_{z} \cdot e^{j \varpi \frac{z}{c}} \mathrm{~d} z\right| & & \text { is the single cell energy gain } \\
\mathrm{E}_{z} & & \text { is the complex accelereting field on axis } \\
\mathrm{E}_{\mathrm{acc}}=\frac{V_{z}}{D}=\frac{\left|\int_{0}^{D} \mathrm{E}_{z} \cdot e^{j \varpi \frac{z}{c} \mathrm{~d} z}\right|}{D} & & \text { is the accelerating field in the cell } \\
\mathrm{P}_{\mathrm{in}}=\int_{S} \frac{1}{2} \operatorname{Re}\left(\underline{E} \times \underline{H}^{*}\right) \cdot \hat{z} \mathrm{~d} S & & \text { is the average input power (flux power) } \\
\mathrm{P}_{\mathrm{diss}}=\frac{1}{2} R_{S} \int_{S_{\mathrm{ext}}}\left|H_{\mathrm{tan}}\right|^{2} \mathrm{~d} S_{\mathrm{ext}} & & \text { is the average dissipated power per unit length } \\
\mathrm{p}_{\mathrm{diss}}=\frac{\mathrm{P}_{\mathrm{diss}}}{D} & & \text { is the stored energy in the cell } \\
W & =\frac{1}{4} \mu_{0} \int_{V} \underline{H} \cdot \underline{H}^{*} \mathrm{~d} V+\frac{1}{4} \varepsilon_{0} \int_{V} \underline{E} \cdot \underline{E}^{*} \mathrm{~d} V \\
w & =\frac{W}{D} & & \text { is the average stored energy per unit length }
\end{array}
$$

The series impedance, field attenuation, group velocity and the ratio between the maximum surface electric field and the accelerating one are shownin figure 18 as a function of the irises half 


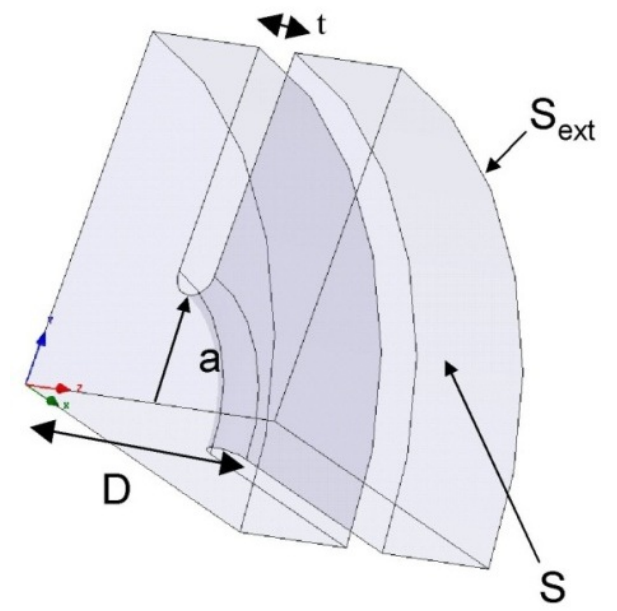

Figure 17. Sketch of the single cell with main parameters.
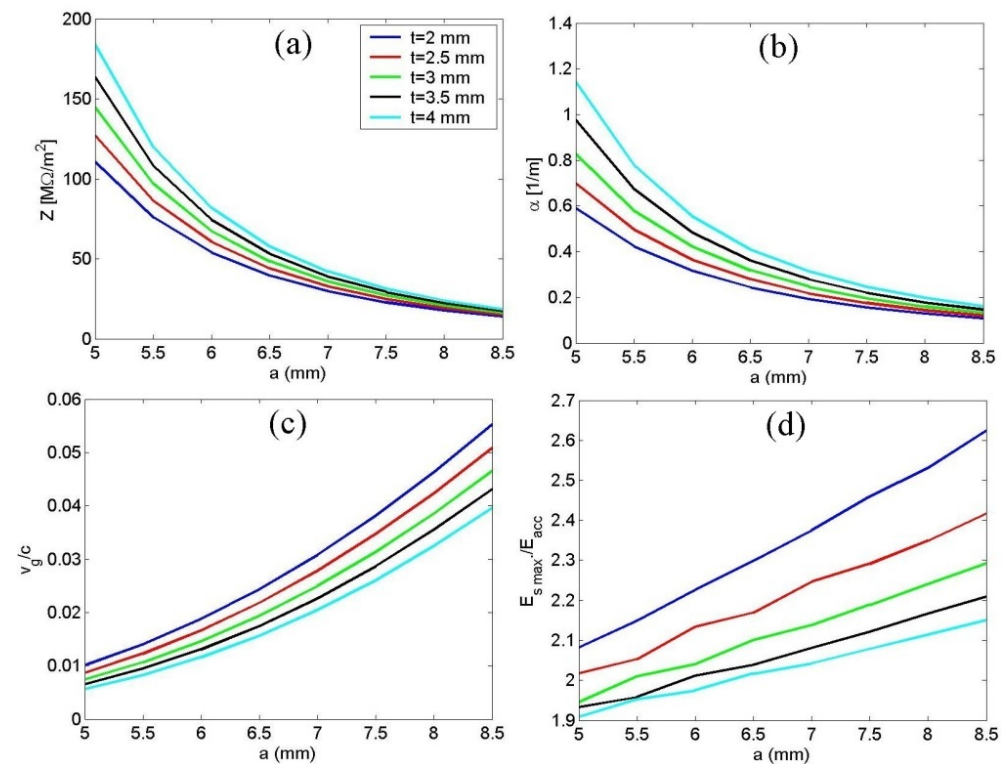

Figure 18. Single cell parameters as a function of the iris dimension and thickness: (a) series impedance; (b) field attenuation constant; (c) group velocity; (d) maximum surface electric field normalized to the accelerating field.

aperture (a) and thickness ( $\mathrm{t}$ ). They have been found using the 2D electromagnetic code SUPERFISH [35] and the procedure illustrated in [36, 37] to calculate the TW parameters from standing wave simulations. In particular the use of the 2D code SUPERFISH allowed a better calculation [37] of the surface electric field on the iris with respect to 3D codes like HFSS.

Figure 19 shows the ratio $\mathrm{E}_{\mathrm{S}_{-} \max } / \mathrm{E}_{\mathrm{acc}}$ as a function of the iris ellipticity for the iris values $\mathrm{a}=7$ $\mathrm{mm}$ and $\mathrm{t}=2.5 \mathrm{~mm}$. Similar results have been obtained for other iris apertures. The plot shows that for a certain iris aperture there is an optimum elliptical profile of the iris itself that minimizes the surface field. The maximum reduction of the surface field is of the order of $8 \%$ with respect to the round profile. 

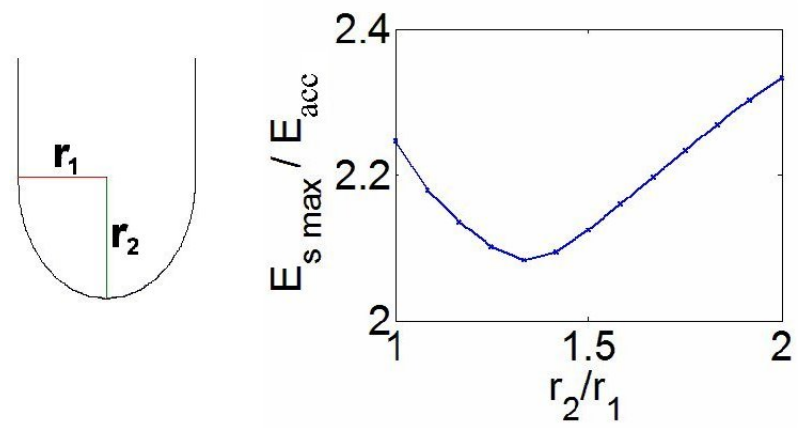

Figure 19. Maximum surface electric field normalized to the average accelerating field as a function of the iris ellipticity (case: $\mathrm{a}=7 \mathrm{~mm}$ and $\mathrm{t}=2.5 \mathrm{~mm}$ ).

\section{B Calculation of the structure parameters with a SLED-type input pulse}

The peak power from the SLED is given by the following formula [38]:

$$
\mathrm{P}_{\text {SLEDout }}(t)=\mathrm{P}_{\text {KLYout }}[\underbrace{\frac{2 \beta}{1+\beta}\left(2-e^{-t_{1} / \tau}\right)}_{G}-\underbrace{\frac{\beta-1}{\beta+1}}_{A}]^{2}=\mathrm{P}_{\text {KLYout }}\left[G e^{-t / \tau}-A\right]^{2}
$$

where $\mathrm{P}_{\mathrm{KLY} \text { out }}$ is the output power from the klystron, $t_{1}$ is the time of the phase switch, $\mathrm{G}$ is the so called SLED gain, $\tau=2 Q_{L} / \omega_{\mathrm{RF}}$ is the decay time, $\beta$ is the coupling coefficient of the SLED cavity, $Q_{L}=Q_{0} /(1+\beta)$ is the loaded quality factor with $Q_{0}$ the unloaded quality factor.

In our particular case the SLED feeds two sections and therefore the input power into a single TW structure as a function of time is:

$$
\mathrm{P}_{\mathrm{TW}}(t)=\frac{\mathrm{P}_{\text {SLEDout }}(t)}{2}=\underbrace{\frac{\mathrm{P}_{\mathrm{KLYout}}}{2}}_{\mathrm{P}_{\mathrm{TW} \_0}}\left[G e^{-t / \tau}-A\right]^{2}
$$

The power flux along a CI TW structure as a function of the longitudinal coordinate $(z)$ and time $(t)$ is then:

$$
\begin{cases}\mathrm{P}_{\mathrm{TW}}(z, t)=\mathrm{P}_{\mathrm{TW}}\left(t-z / v_{g}\right) e^{-2 \alpha z} & z \leq \mathrm{tv}_{g} \\ \mathrm{P}_{\mathrm{TW}}(z, t)=0 & z>\mathrm{tv}_{g}\end{cases}
$$

The corresponding accelerating field is given by:

$$
\begin{cases}\mathrm{E}_{\mathrm{acc}}(z, t)=\sqrt{\mathrm{ZP}_{\mathrm{TW}}\left(t-z / v_{g}\right)} e^{-\alpha z}=\sqrt{\mathrm{ZP}_{\mathrm{TW} \_0}}\left(G e^{-\frac{t-z / v_{g}}{\tau}}-A\right) e^{-\alpha z} & z \leq \operatorname{tv}_{g} \\ \mathrm{E}_{\mathrm{acc}}(z, t)=0 & z>\mathrm{tv}_{g}\end{cases}
$$

Based on the facts that: a) the peak surface field occurs at the entrance of structures and, b) the maximum power is given at the beginning of the input pulse from the ratio $\mathrm{E}_{\mathrm{s} \text {-max }} / \mathrm{E}_{\mathrm{acc}}$ (see figure 18(d)) the maximum surface field is simply given by:

$$
\mathrm{E}_{\mathrm{s} \_ \text {peak }}=\left(\frac{\mathrm{E}_{\mathrm{s} \_ \text {max }}}{\mathrm{E}_{\mathrm{acc}}}\right) \mathrm{E}_{\mathrm{acc}}(0,0)=\left(\frac{\mathrm{E}_{\mathrm{s}_{\mathrm{max}} \max }}{\mathrm{E}_{\mathrm{acc}}}\right) \sqrt{\mathrm{ZP}_{\mathrm{TW}_{-} 0}}(G-A)
$$


while the accelerating field distribution after one filling time $\tau_{\mathrm{F}}=L / v_{g}$ is:

$$
\mathrm{E}_{\mathrm{acc}}(z)=\mathrm{E}_{\mathrm{acc}}\left(z, t=L / v_{g}\right)=\sqrt{\mathrm{ZP}_{\mathrm{TW} \_0}}\left(G e^{\frac{z-L}{v_{g} \tau}}-A\right) e^{-\alpha z}
$$

where $L$ is the structure length.

The total beam energy gain in the structure $\left(\mathrm{eV}_{\mathrm{TOT}}\right)$ and the average accelerating field can be calculated from eq. (B.6):

$$
\begin{aligned}
& e V_{\mathrm{TOT}}=e \int_{0}^{L} \mathrm{E}_{\mathrm{acc}} \mathrm{d} z=e \sqrt{\mathrm{ZP}_{\mathrm{TW}_{\_} 0}}\left(G \frac{e^{-\frac{\tau_{\mathrm{F}}}{\tau}}-e^{-\alpha L}}{\alpha-\frac{1}{v_{g} \tau}}-A \frac{1-e^{-\alpha L}}{\alpha}\right) \\
& \mathrm{E}_{\text {acc_av }}=\frac{V_{\mathrm{TOT}}}{L}
\end{aligned}
$$

The factor $\left(G e^{\frac{z-L}{v_{g} \tau}}-A\right) e^{-\alpha z}$ in eq. (B.6) can never be constant with $z$. Therefore, even if there is some field attenuation along the CI structure, it is not possible to compensate the typical SKIP RF pulse with the field attenuation intrinsic to the CI structure and obtain a perfectly uniform accelerating field along the structure. Nevertheless the non-uniformity of the field between the initial and final parts of the structure is significantly smaller if compared to a constant gradient structure fed by the SLED input pulse. More precisely, the uniformity of the field (FU) can be evaluated with the following ratio:

$$
\mathrm{FU}=\frac{\left|\mathrm{E}_{\mathrm{acc}}(0)-\mathrm{E}_{\mathrm{acc}}(L)\right|}{\left(\mathrm{E}_{\mathrm{acc}}(0)+\mathrm{E}_{\mathrm{acc}}(L)\right) / 2} \Rightarrow 2 \frac{\left|G\left(e^{-\alpha L}-e^{-\frac{\tau_{\mathrm{F}}}{\tau}}\right)+A\left(1-e^{-\alpha L}\right)\right|}{\left|G\left(e^{-\alpha L}+e^{-\frac{\tau_{\mathrm{F}}}{\tau}}\right)-A\left(1+e^{-\alpha L}\right)\right|}
$$

Figure 20 shows the $\mathrm{FU}$ as a function of the group velocity for a $\mathrm{C}$ Band section with the following typical parameters of a CI structure and a SKIP (see next section): $L=1.5 \mathrm{~m}, G=3$, $A=0.7, \tau=1 \mu \mathrm{s}$. For a wide range of group velocity near $v_{g} / c \cong 0.02$, the field uniformity FU is below $10 \%$. There is a particular value of $v_{g} / c$ for which FU $=0$. For lower values of $v_{g} / c$, the FU is dominated by the field attenuation parameter and the electric field at the entrance of the structure is higher than at the end.

The average dissipated power into the structure is equal to:

$$
\mathrm{p}_{\mathrm{diss} \_a v} \cong \mathrm{P}_{\mathrm{TW} \_0}\left(1-e^{-2 \alpha L}\right)\left[\frac{G^{2}}{2 \tau}\left(1-e^{-2 t_{\mathrm{SLED}}}\right)-\frac{2 A G}{\tau}\left(1-e^{-t_{\mathrm{SLED}}}\right)+A^{2} t_{\mathrm{SLED}}\right] f_{\text {rep }}
$$

where $t_{\mathrm{SLED}}$ is the duration of the peak SLED pulse and $f_{\text {rep }}$ is the repetition frequency of the pulses (in general the parameters are chosen in order to have $t_{\mathrm{SLED}}=\tau_{\mathrm{F}}$ ). Eq. (B.10) does not include the contribution of the pre-pulse of SLED, negligible.

\section{Analysis of the multipolar field components introduced by the couplers}

The coupler is a compact transition between a rectangular geometry (the waveguide) and a circular one and, therefore, it introduces multi-polar field components in the waveguide region. Due to the symmetric feed of the system these components have an even periodicity with respect to the 


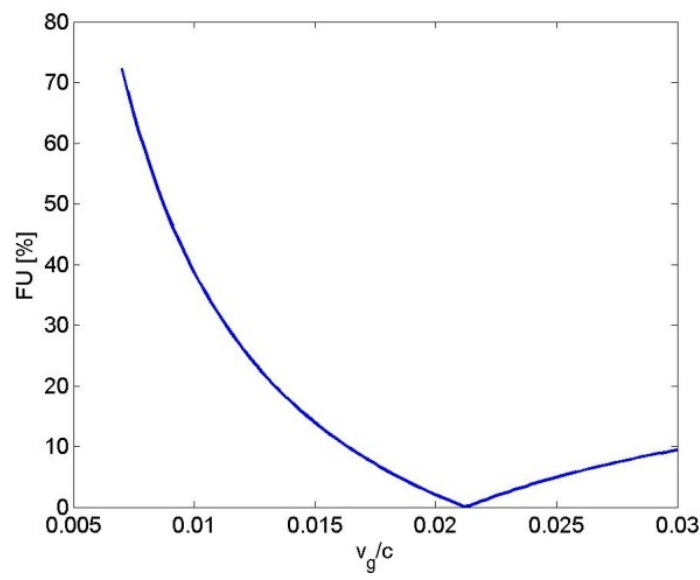

Figure 20. FU as a function of the group velocity for a $\mathrm{C}$ Band section with the following typical parameters (see next section): $L=1.5 \mathrm{~m}, G=3, A=0.7, \tau=1 \mu \mathrm{s}$.

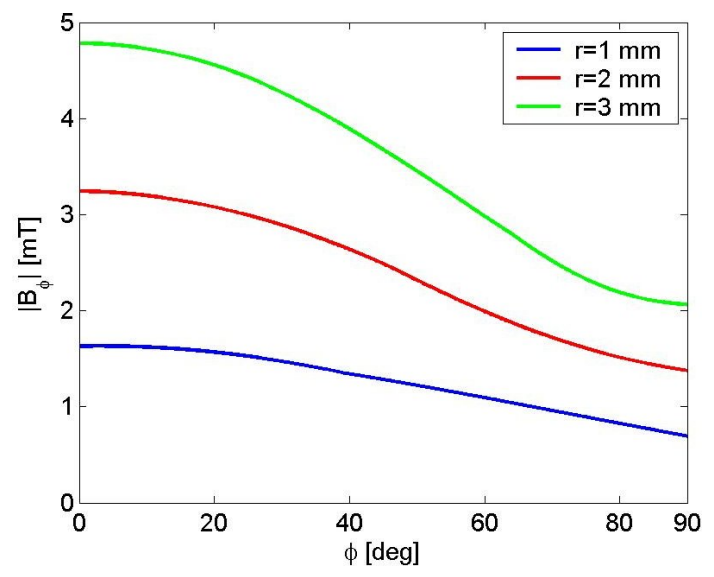

Figure 21. Azimuthal magnetic field in the center of the waveguide coupler calculated for three different circle radii.

azimuthal angle. More precisely the azimuthal magnetic field can be developed to first order as follows:

$$
B_{\varphi}(r, \varphi, z) \cong A_{0}(z) r+\sum_{n=1}^{\infty} A_{2 n}(z) \cos (2 n \varphi) r^{2 n-1}
$$

where the $A_{2 n}$ components are complex functions and depend on the longitudinal coordinate $z$.

The quadrupolar component is the component associated to the term with $n=1$ and the gradient of the quadrupole component is exactly the term $A_{2}$.

Figure 21 illustrates the azimuthal magnetic field in the center of the waveguide coupler for three different circle radii. The calculation was performed for an average accelerating field of 35 $\mathrm{MV} / \mathrm{m}$ along the structure. The field profile is mainly driven by the quadrupolar component $(2 \varphi$ dependence), but shows other high order components.

In principle it is possible to calculate all multi-polar components at different longitudinal coordinates by calculating the Fourier series components in different arcs. As a first order we have 


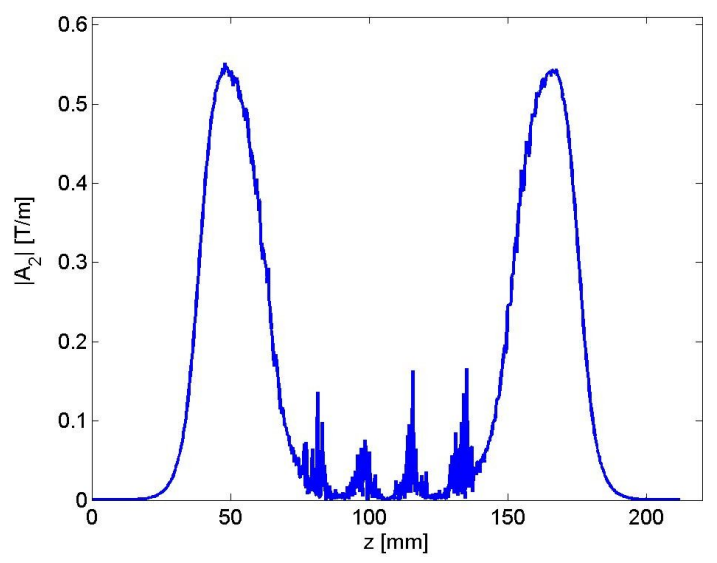

Figure 22. Absolute value of the quadrupole gradient calculated using equation (B.10) for $\mathrm{r}_{0}=3 \mathrm{~mm}$.

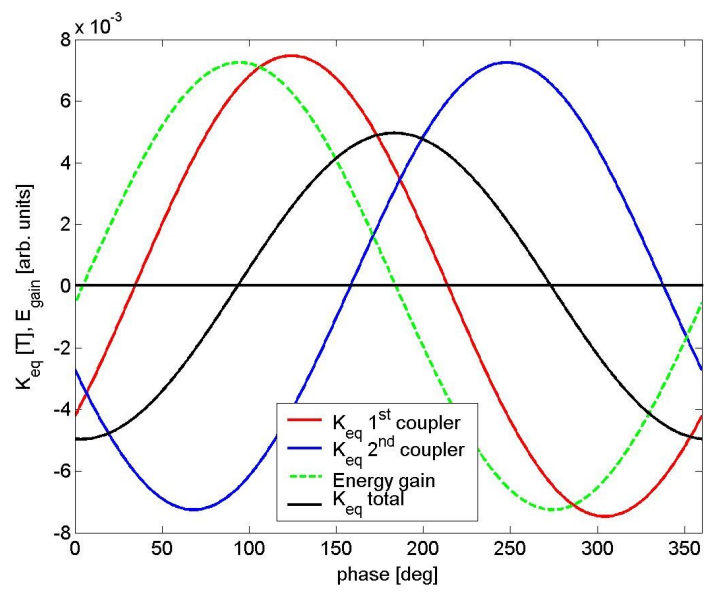

Figure 23. Integrated quadrupole gradient in the first and second coupler $\left(K_{\mathrm{eq}}\right)$ as a function of the injection phase.

estimated the quadrupole gradient along the structure in the following way:

$$
A_{2}(z) \cong \frac{B_{\max }\left(r_{0}, z\right)-B_{\min }\left(r_{0}, z\right)}{2 r_{0}}
$$

where $B_{\max }$ and $B_{\min }$ are the maximum and minimum of the magnetic field at a certain distance $r_{0}$ from the axis and at a given longitudinal coordinate $z$. Figure 22 shows the absolute value of the quadrupole gradient for $r=3 \mathrm{~mm}$. The integrated quadrupole gradient in the first and second coupler $\left(K_{\text {eq }}\right)$ as a function of the injection phase is shown in figure 23. In the same figure we have plotted the energy gain. The sum of the two integrated quadrupole components shows a $90^{\circ}$ difference with respect to the accelerating field.

All these results show that this quadrupole gradient is small if compared to usual magnetic quadrupoles but a further investigation of the effects on the beam dynamics have to be done. In particular figure 23 shows that this quadrupole component varies along the bunch.

The quadrupole component can be minimized [37] by modifying the geometry of the coupler as shown in figure 24. The introduced bump changes this quadrupole amplitude and a minimum 

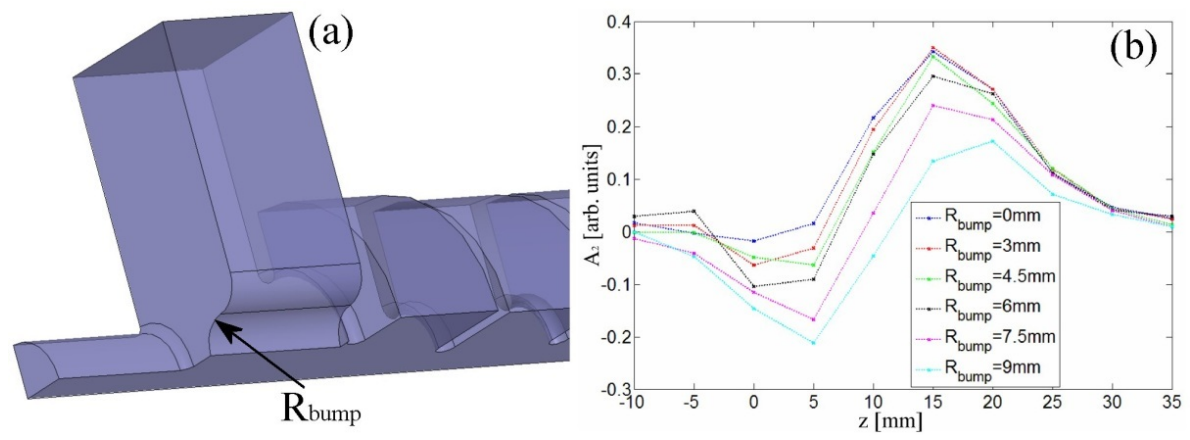

Figure 24. Bump on the waveguide coupler to minimize the quadrupole component of the field.

contribution (at least one order of magnitude smaller than the shown one) can be obtained.

We have also calculated the dipole components that can be excited in the input coupler because of the unbalances between the phase of the two harms of the integrated splitter. The result is the following:

$$
V_{T}[\mathrm{kV}] \cong 0.16 \cdot \Delta \phi[\mathrm{deg}] \cdot \sqrt{\mathrm{P}_{\min }[\mathrm{MW}]}
$$

Where $V_{T}$ is the integrated transverse kick, $\Delta \phi$ is the phase difference between the two harms of the splitter and $\mathrm{P}_{\text {in }}$ is the input power. The formula (C.3) has been obtained by HFSS simulation. From the formula it is clear that if the phase unbalance is within few degree the transverse kick is negligible.

\section{References}

[1] SCSS X-FEL R\&D Group, SCSS X-FEL conceptual design report, T. Shintake and T. Tanaka ed., RIKEN Harima Institute/SPring-8, Sayo Japan (2004).

[2] T. Shintake et al., Stable operation of a self-amplified spontaneous-emission free-electron laser in the extremely ultraviolet region, Phys. Rev. Spec. Top. AC 12 (2009) 070701.

[3] T. Shintake et al., Experiment of nanometer spot size monitor at FFTB using laser interferometry, in proceedings of the Particle Accelerator Conference (PAC95), May 1-5, Dallas, Texas U.S.A. (1995).

[4] T. Shintake, The choke mode cavity, Jpn. J. Appl. Phys. 31 (1992) L1567.

[5] R.Ganter et al., SwissFEL conceptual design report, Report 10-04 (2010).

[6] R. Zennaro et al., Conceptual design of the C-Band module for the SwissFEL, in the proceedings of the XXV Linear Accelerator Conference (LINAC10), September 12-17, Tsukuba, Japan (2010).

[7] K. Akai et al., Design progress and construction status of SuperKEKB, talk given at the International Particle Accelerator Conference 2012 (IPAC2012), May 20-25, New Orleans, Louisiana, U.S.A. (2012).

[8] S. Fukuda, et al., Linac upgrade plan using a C-band system for SuperKEKB, talk given at the $30^{\text {th }}$ Advanced ICFA Beam Dynamics Workshop on High Luminosity $e^{+} e^{-}$Collisions, October 13-16, Stanford, California, U.S.A. (2003).

[9] T. Shintake, HOM-free linear accelerator structure using choke mode cavity, in the proceedings of the $17^{\text {th }}$ Linear Accelerator Meeting, September 1-3, Sendai, Japan (1992), KEK Report No. 92-66. 
[10] T. Shintake, Design of high power model of damped linear accelerating structure using choke mode cavity, in the proceedings of the Particle Accelerator Conference (PAC93), May 17-20, Washington, U.S.A. (1993).

[11] T. Shintake et al., High-precision spot size monitors for $e^{+} e^{-}$linear colliders, in the proceedings of the $17^{\text {th }}$ International Linear Accelerator Conference (LINAC94), August 21-26, Tsukuba, Japan (1994).

[12] H. Matsumoto et al., Low temperature brazing technique for accelerators, in the proceedings of the $5^{\text {th }}$ European Particle Accelerator Conference (EPAC96), June 10-14, Sitges, Barcelona, Spain (1996).

[13] H. Matsumoto et al., Fabrication of the C-Band $5712 \mathrm{MHz}$ choke-mode type damped accelerator structure, in the proceedings of XIX International Linac Conference (LINAC98), August 24-28, Chicago, Illinois, U.S.A. (1998).

[14] T. Shintake et al., The first wakefield test on the C-band choke-mode accelerating structure, in the proceedings of the Particle Accelerator Conference (PAC99), March 29-April 2, New York, U.S.A. (1999).

[15] T. Inagaki, 8-GeV C-Band accelerator construction for XFEL/SPring-8, in the proceedings of the XXIV Linear Accelerator Conference (LINAC08), September 29-October 3, Victoria, Canada (2008).

[16] D. Alesini at al., Beam energy upgrade of the Frascati FEL LINAC with a C-band RF system, in the proceedings of $1^{\text {st }}$ International Particle Accelerator Conference (IPAC10), May 23-28, Kyoto, Japan (2010).

[17] V. Fusco and M. Ferrario, Beam dynamics study of a C-band LINAC driven FEL with S-band photo-injector, in the proceedings of the Particle Accelerator Conference (PAC09), May 4-8, Vancouver, Canada (2009).

[18] D. Alesini et al., The SPARC project: a high-brightness electron beam source at LNF to drive a SASE-FEL experiment, Nucl. Instrum. Meth. A 507 (2003) 345.

[19] M. Ferrario et al., Velocity bunching experiments at SPARC, in the proceedings of the Particle Accelerator Conference (PAC09), May 4-8, Vancouver, Canada (2009).

[20] M. Ferrario et al., Experimental demonstration of emittance compensation with velocity bunching, Phys. Rev. Lett. 104 (2010) 054801.

[21] M. Labat et al., High-gain harmonic-generation free-electron laser seeded by harmonics generated in gas, Phys. Rev. Lett. 107 (2011) 224801.

[22] D. Palmer, The next generation photoinjector, Ph.D. thesis, Stanford University, U.S.A. (1998).

[23] T. Sugimura, SKIP - A pulse compressor for SuperKEKB, in the proceedings of XXII Linear Accelerator Conference 2010 (LINAC04), August 16-20, Lübeck, Germany (2004).

[24] D. Alesini et al., Design, realization and low power rf tests of the C band structure prototype for SPARC, SPARC-RF-11/002 (2011).

[25] V.A. Dolgashev, et al., RF breakdown in normal conducting single-cell structures, in the proceedings of Particle Accelerator Conference (PAC05), May 16-20, Knoxville, Tennessee, U.S.A. (2005).

[26] C. Nantista, S. Tantawi and V. Dolgashev, Low-field accelerator structure couplers and design techniques, Phys. Rerv. ST Accel. Beams 7 (2004) 072001.

[27] V.A. Dolgashev, High magnetic fields in couplers of X-band accelerating structures, in the proceedings of the Particle Accelerator Conference (PAC03), May 12-16, Portland, U.S.A. (2003). 
[28] D. Alesini, Power Coupling, CERN Yellow Report CERN-2011-007 (2011) [arXiv:1112.3201].

[29] D. Alesini et al., Design of couplers for traveling wave RF structures using 3D electromagnetic codes in the frequency domain accoppiatori, Nucl. Instrum. Meth. A 580 (2007) 1176.

[30] Comeb website, www.comeb.it

[31] C. Steele, A nonresonant perturbation theory, IEEE T. Microw. Theory 14 (1966) 70.

[32] J. Shi et al., Tuning of clic accelerating structure prototypes at CERN, CERN-ATS-2010-210 (2010).

[33] G. Campogiani, Tuning of a C-Band accelerating cavity, Degree Thesis, University of Rome "La Sapienza", Rome, Italy (2011).

[34] D. Alesini et al., High-gradient test of C-band accelerating structure prototype for energy upgrade of frascati photoinjector SPARC, SPARC-RF-11-005 (2011).

[35] Poisson Superfish website, http://laacg1.lanl.gov/laacg/services/download_sf.phtm.

[36] G.A. Loew et al., Computer calculations for travelling wave periodic structure properties, IEEE Trans. Nucl. Sci. NS-26 (1979) 3701.

[37] V. Spizzo, Progetto elettromagnetico di una struttura accelerante ad onda viaggiante in banda C, master degree thesis, University of Rome "La Sapienza", Rome, Italy (2009).

[38] D. Farkas et al., SLED: a method of doubling SLAC's energy, SLAC-PUB-1453 (1974).

[39] R. Zennaro, PSI activities/plans, talk given at the CLIC workshop 2013, January 28-February 1, CERN, Switzerland (2013).

[40] A. Grudiev, S. Calatroni and W. Wuensch, A new local field quantity describing the high gradient limit of accelerating structures, Phys. Rev. ST Accel. Beams 12 (2009) 102001. 